\title{
Efficient Contention Resolution in Highly Dense LTE Networks for Machine Type Communications
}

\author{
Andres Laya*, Luis Alonso ${ }^{\dagger}$ and Jesus Alonso-Zarate ${ }^{\ddagger}$ \\ *KTH Royal Institute of Technology, Sweden. e-mail: laya@kth.se \\ ${ }^{\dagger}$ Universitat Politècnica de Catalunya (UPC), Spain. e-mail: luisg@ @tsc.upc.edu \\ ${ }^{\ddagger}$ Centre Tecnològic de Telecomunicacions de Catalunya (CTTC), Spain. e-mail: jesus.alonso@cttc.es
}

\begin{abstract}
In this paper, we propose a solution to efficiently handle the initial access contention for highly dense LTE networks, which still represents a challenge in terms of the high number of devices associated to Machine Type Communications. The analysis is based on the fact that as the radius of a LTE cell is larger, the number of available preambles generated from a single root sequence is reduced. The current access mechanism, based on frame-slotted ALOHA, does not operate efficiently under these conditions, negatively affecting the access delay and collision probability. We show how can the access procedure be improved by implementing tree-splitting algorithms in order to cope with high number of simultaneous arrivals in large cells, above $5 \mathrm{~km}$ in radius. The implementation achieves as much as $85 \%$ reduction on the access delay and $54 \%$ reduction on energy consumption for cells with radius larger than $39 \mathrm{~km}$.
\end{abstract}

Index Terms-Machine Type Communications; Energy consumption; LTE-Advanced; Machine-to-Machine communications; Tree-splitting; Distributed Queuing.

\section{INTRODUCTION}

Machine-to-Machine (M2M) communications or Machine Type Communication (MTC), in 3GPP terminology, impose several challenges on cellular networks [1]. Among these, 3GPP has addressed over a technical report the need to design improvements for the access mechanisms of cellular systems to be able to handle applications where the number of devices raises up to tens of thousands per cell. The smart meters case presented by the 3GPP in [2], [3] is based on the scenario of a urban area, where a single cell could cover up to 35000 households, if these households are to be equipped with 3 smart meters each (electricity, gas and water), it sums up to 105000 devices in a cell. There are other applications, such as earthquake warning, fire warning and synchronization after major power outage, in which thousands of MTC devices would attempt to access the network based on the same triggering event, within a very short time frame. This report resulted in the inclusion of the Access Class Barring (ACB) scheme as part of the Release 8 and Extended Access Barring (EAB) in Release 11 to avoid MTC overload. The main limitation of these solutions is that they are based on long backoff periods to disperse access attempts, which may have a negative impact on battery-operated device. This is the motivation of the work presented in this paper, where we focus on the initial access on LTE-A networks.

The queuing theory analysis presented in [4] shows that LTE-A system is not stable when the occupation rate is high. In order to overcome these limitations, many schemes have been proposed to manage the congestion based on large number of MTC devices, as compiled in [5]. However, these proposals fall sort on providing a feasible solution with a considerate balance between access delay, access probability rate and energy consumption. Moreover, the fact that the number of orthogonal preambles available for contention decreases as the cell radius increases has not been considered and this relation imposes an additional limitation for the current approach.

There exist a particular approach that can tackle the instability issue in a more efficient manner. Campbell and $\mathrm{Xu}$ [6] proposed a MAC protocol whose high performance is completely independent of the number of devices sharing a common channel, referred to as Distributed Queuing (DQ). This is specially fitted to the high density of devices in MTC. Since the first proposal [6], several studies have analyzed the performance of the protocol [7], [8], demonstrating the stability of its performance and the near optimum behavior in terms of channel utilization, access delay, and energy consumption for all system layouts. Furthermore, several extensions and adaptations have been also proposed such as for $3 \mathrm{G}$ networks [9], WLAN [10], mobile ad-hoc networks [11] and BANs [12].

The DQ principles cannot be directly applied to LTE and modifications are required to implement them with minimal alterations to the standards. This is the contribution of this paper, where we propose the use of DQ mechanisms as the most feasible solution to improve the contention-based Random Access (RA) procedure, used for initial association of many uncoordinated devices in LTE-A, regardless of the cell size. By means of simulations, we achieve as much as $85 \%$ reduction on the access delay and 54\% reduction on energy consumption for cells with radius larger than $39 \mathrm{~km}$, while maintaining the blocking probability to a minimum.

The organization of the document is the following: on Section II, the access mechanisms used in LTE-A are explained, providing an overview of the RA procedure in Section II-A, followed by an explanation of the preamble sequence generation in Section II-B. These to concepts are required in order to understand the relation between the cell size and the resources available for contention, as presented in Section II-C. In Section III, we provide a general introduction to the DQ concept. The integration of DQ into the RA procedure is given in Section IV. In Section V-A, we present the system and simulation setup that we use to obtain the comparative results presented in Section V-B. Finally, the conclusions and further remarks are provided in Section VI. 


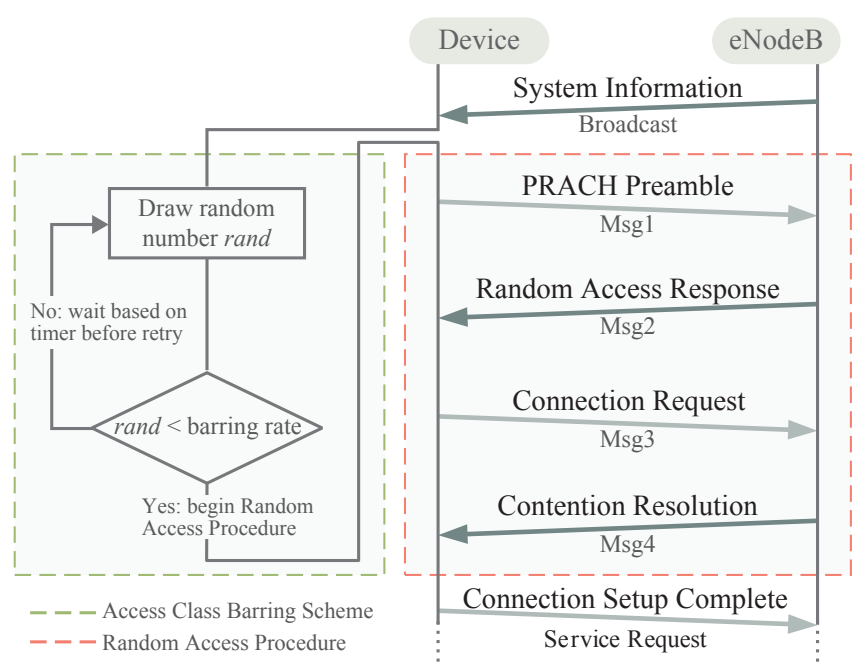

Fig. 1. Access Class Barring (ACB) scheme and contention-based Random Access (RA) Procedure in LTE-Advanced.

\section{ACCESs Mechanisms In LTE-A}

In this section, the RA procedure and RA preambles are described, followed by an explanation of the relation between the cell size and the resources available for contention.

\section{A. Overview of the LTE-A random access procedure}

The RA procedure occurs in the following cases [5], [13]:

1) Upon initial network access, i.e., association process.

2) Synchronization for new data transmission or reception.

3) Upon transmission of new data when no scheduling request resources are configured.

4) In the case of handover.

5) After radio link failures, for connection re-establishment. The RA procedure can be contention-based or contentionfree. The former can be used in all the previously listed cases and it is prone to collision. The latter is used in case of downlink data arrival and handover, for these cases, the eNodeB is able to prevent collisions [5]. This study is based on the contention-based RA procedure, which consists on a four-message handshake between the device and the eNodeB. As shown in Fig. 1, these messages are:

MSG. 1, RA PREAMBLE: it is a signature each device use when attempting an access. It is constructed on 6 bits, giving a maximum of 64 possibilities. For contention-based access, a device randomly selects a preamble and transmits it on a specific resource in the Random Access CHannel (RACH). The RACH is formed by a periodic sequence of allocated timefrequency resources, referred to as RA slots. In LTE Frequency Division Duplex (FDD), the RA slot periodicity varies between 1 and $20 \mathrm{~ms}$. [14]. If more than one device sends the same preamble over the same RA slot, a collision occurs and the feedback is sent by the eNodeB on Msg. 2.

MsG. 2, RANDOM ACCESS RESPONSE (RAR): if the eNodeB detects a preamble, it replies with the RAR. This message is sent over the Physical Downlink Shared CHannel $(\mathrm{PDSCH})$ and contains the following information:

- Identity of the detected preamble.

- Timing alignment instructions for uplink synchronization.
- Initial uplink resource grant, to be used in Msg. 3.

- A Cell Radio Network Temporary Identifier (C-RNTI).

- An optional Backoff Indicator (BI).

The RAR contains different subheaders, one for the BI information and additional subheaders to deliver information to each detected preamble. If a device receives a RAR without information for the preamble it used, it will perform a backoff time according to the BI parameter [15].

If multiple devices sent the same preamble over the same RA slot, the eNodeB might detect the collision based on the difference in transmission delay and it will not provide a RAR. However, if the devices are at the same distance from the eNodeB, the collision might be undetected and the same RAR information will be decoded by more than one device. For these cases, the contention will be solved on Msg. 3 .

MsG. 3, Connection ReQuest: after the initial uplink resource grant informed in Msg. 2, the device transmits a connection request to the eNodeB. This information conveys the device identifier (C-RNTI) and the establishment cause. In case of undetected preamble collision, more than one device was assigned with the same uplink resource; the eNodeB will detect the Msg. 3 collision and it will not acknowledge this message; resulting on access failure for the devices.

MsG. 4, Contention Resolution: a device receiving Msg. 4 will have a successful access. If there is no successful reception, a new access attempt is scheduled.

The 3GPP included the Access Class Barring (ACB) scheme to provide an additional control mechanism and prevent access failures [2]. The device determines the barring status with the information provided from the serving network. If the ACB is active, the device draws a uniform random number between 0 and 1 when initiating connection establishment and compares with the current barring rate to determine whether it is barred, as shown in Fig. 1. The barring factor ranges from 0 to $95 \%$ and the barring time spans from 4 to 512 seconds [14].

\section{B. Random Access Preamble}

The 64 different preamble sequences available per cell are constructed from one or several root Zadoff-Chu (ZC) sequences $^{1}$ and their cyclic shifts $N_{C S}$ [18]. A total of 838 root sequences were defined for LTE [19].

Two main parts compose the preamble, a Cyclic Prefix (CP) and a preamble sequence, as shown in Fig. 2. The CP corresponds to the end of the sequence, which is attached at the start of the preamble to enable a periodic correlation at the PRACH receiver [13]. Four preamble formats are defined for LTE FDD operation and they differ in the length of the preamble, as specified in [18] and analyzed in [13], [19]. The preamble length has been designed to be shorter than the PRACH slot to provide a Guard Time (GT) and absorb the propagation delay. The length of the $\mathrm{CP}$ and the GT shall cover the maximum Round Trip Delay (RTD). The lower side

\footnotetext{
${ }^{1}$ Zadoff-Chu (ZC) sequences were first published in [16], [17]. These sequences satisfy a Constant Amplitude Zero Autocorrelation property, which means that orthogonal sequences can be generated from the same $\mathrm{ZC}$ root by means of cyclic shifts [13]
} 


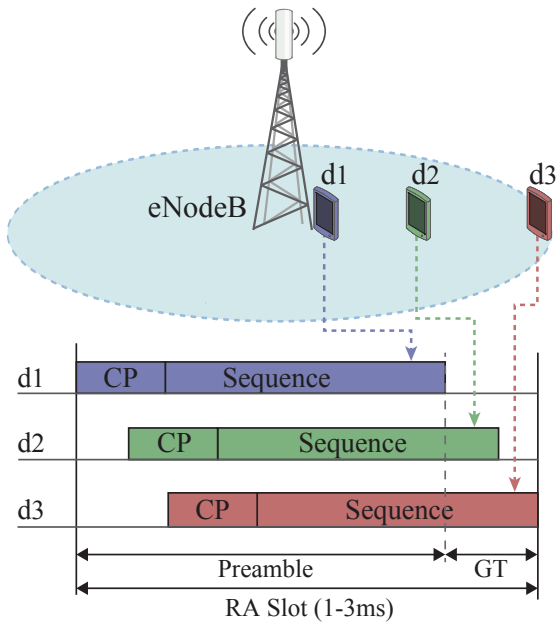

Fig. 2. Preamble sequence and guard time. The top side of the figure shows the location of a terminal in relation to the eNodeB. The lower side of the figure shows the received preambles at the eNodeB.

of Fig. 2 shows three preambles at the receiver with different timings due to the variation in their propagation delay [13].

In the next section, the relation between the cell size and the number of orthogonal preambles will be detailed.

\section{Cell size and available orthogonal preambles}

The duration of the preamble sequence has been designed to maximize the number of orthogonal preambles. Even though longer sequences could improve the coverage and increase the number of orthogonal preambles from the same sequence, they require longer $\mathrm{CP}$ and GT in order to cope with the corresponding Round Trip Delay (RTD). This also means that the lengths of the CP and GT can be estimated based on the maximum RTD of the sequence [13]. Therefore, the format selection is tightly bounded to the cell radius, i.e., the larger the cell-size, the larger the preamble length should be. The cell radius can be calculated based on the GT, which should be as minimum equal to the time of the RTD $T_{R T D}$ (Eq.1).

$$
T_{R T D}=\frac{2 \times \text { Cellradius }}{c}
$$

The $T_{R T D}$, the preamble sequence length $T_{S E Q}$ (which correspond to $800 \mu \mathrm{s}$ in LTE) and the length of the ZC sequence $N_{Z C}$ (which correspond to 839 samples in LTE) determine the minimum length of the cyclic shift duration $N_{C S}$ in terms of number of samples, following Eq.2.

$$
N_{C S}=\left\lceil\frac{T_{R T D}+T_{\text {delayspread }}}{T_{S E Q}} N_{Z C}\right\rceil
$$

The maximum number of preambles $N_{\text {Preambles }}$ which can be constructed from one root sequence is defined by Eq.3.

$$
N_{\text {Preamble }}=\left\lfloor\frac{N_{Z C}}{N_{C S}}\right\rfloor
$$

The number of ZC root sequences required to produce 64 preambles in a single cell is determined by Eq.4.

$$
N_{\text {rootsequence }}=\left\lceil\frac{64}{N_{\text {Preamble }}}\right\rceil
$$

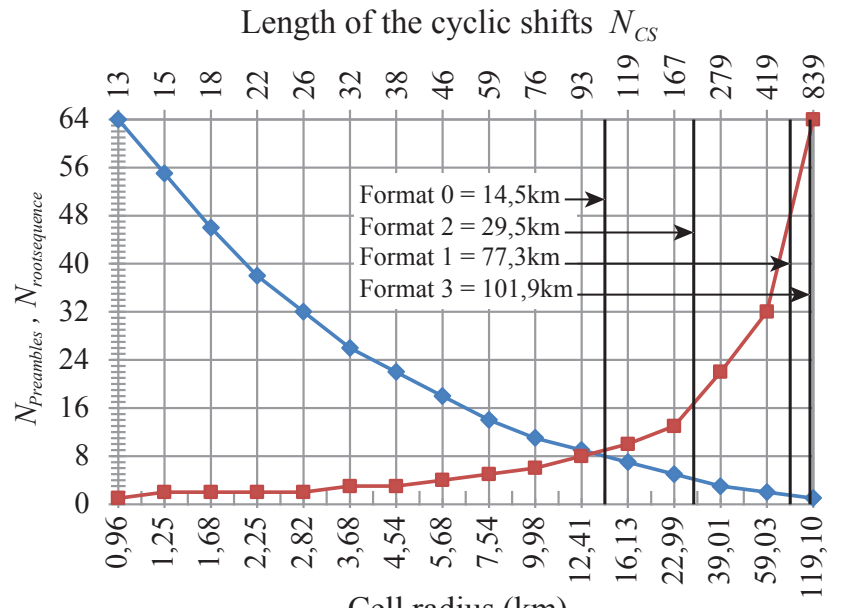

Cell radius $(\mathrm{km})$

$\longrightarrow$ No. of preambles $N_{\text {Preambles }} \quad \longrightarrow$ No. of root sequences $N_{r}$

Fig. 3. Relationship between the Number of available orthogonal preambles $N_{\text {Preambles }}$, the length of the cyclic shifts $N_{C S}$, the cell radius and the required number of $\mathrm{ZC}$ root sequences $N_{\text {rootsequence. }}$

According to the procedure described above, Fig. 3 shows that all 64 preamble sequences can be generated form a single root sequence only if the cell radius is shorter than $958 \mathrm{~m}$. Following the same reasoning, for a cell radius larger than $59 \mathrm{~km}$, each preamble sequence must be generated from a different root sequence. In short, the zero cross-correlation property of the root sequence only exists among shifts of the same root, and using the same root for all preambles results in less interference. Therefore, the RACH is more prone to suffer from congestion in cases related to widespread deployment of sensors in a area covered by a single macro cell. For this reason, it is desirable to present a proposal that can handle more access with fewer resources.

\section{Distributed Queuing FOR CONTENTION RESOLUTION}

The Distributed Queuing (DQ) paradigm is based on the combination of a m-ary tree splitting algorithm with a set of rules that allow organizing devices in virtual queues. The devices are split into groups, reducing the probability of collision due to simultaneous transmission attempts. The queues are distributed in the sense that they have partial representation at each device using counters to represent the queue length and the current position of the device within the queue. The values of each counter are updated autonomously, allowing devices to keep a status with the position in the queue. In this way, the devices can process their transmission turn, acquiring the access grant for transmission.

The distributed scheduling of the queues permits having almost full channel utilization regardless of its capacity, the number of the transmitting devices, and the traffic pattern. Due to the rules of DQ, it behaves as a random access method for low traffic loads, and it switches smoothly and seamlessly to a reservation access method as the traffic load increases. Previous work found in the literature review have proven that using 3 minislots for a preamble transmission in DQ systems 
provides the optimum performance [7], [8]. The interesting factor is that previous adaptation for wireless technologies did not count with a system capable to provide orthogonal preambles. Leveraging on the availability of these preambles in LTE, instead of using consecutive minislots per frame, different preambles on the same RA Slot are used.

Fig. 4 depicts a representation of the algorithm execution. In the first RA Slot, devices select one preamble to request access. If more than one device selects the same preamble, a RA Slot is assigned exclusively to the set of colliding devices that used the same preamble. These devices enter a queue referred to as Collision Resolution Queue (CQR). For each preamble collision there will be a different contention group and the CRQ length will increase by one. The access point (eNodeB) must provide feedback for the RA Slots status together with the CRQ length so each device can compute its position in the queue. Each device computes a representation of the CRQ by means of two integer numbers:

1) Calculate the CRQ length $(R Q$ counter):

- If there have been collisions pending resolution $(R Q>$ 0 ), reduce $R Q$ by one to account for the resolution attempt of the devices at the head of the CRQ.

- Increase the value of $R Q$ by one for each preamble with a collision state in the previous RA Slot.

2) Calculate the device position in the CRQ ( $p R Q$ counter):

- If the device is waiting in the CRQ $(p R Q>0)$, it must first decrease its $R Q$ and $p R Q$ values by one and then increase the $R Q$ by one for each preamble with a collision state in the previous RA Slot.

- If the device has transmitted an preamble on the previous RA Slot and collided, the device sets its $p R Q$ value to point at $R Q$.

On the example shown in Fig. 4, seven devices transmit a preamble at RA Slot $1 . \mathrm{d} 1, \mathrm{~d} 2, \mathrm{~d} 3$ and $\mathrm{d} 4$ collide with preamble 1 and enter in the first position in the CRQ; d5 succeeds with preamble 2; d6 and d7 collide with preamble 3 and enter in the second position.

At RA Slot 2, d1, d2, d3 and d4 contend since they are at the first position in the CRQ, d6 and d7 will wait in the queue until the next RA Slot. $\mathrm{d} 1$ and $\mathrm{d} 2$ collide again with preamble $2 ; \mathrm{d} 3$ and $\mathrm{d} 4$ collide with preamble 3 . Both groups enter at the end of the CRQ on positions 2 and 3, respectively.

At RA Slot 3, d6 and $\mathrm{d} 7$ are in the first CRQ position so they contend, since each device used a different preamble, both succeed and leave the CRQ. At RA Slot 4, d1 and d2 contend again and succeed. Finally, d4 and d5 succeed at RA Slot 5.

\section{DQ INTEGRATION INTO THE RA PROCEDURE}

\section{A. DQ-based RA procedure}

This approach will treat each RA Slot as a separate resource. It means that devices will select a specific RA Slot for the first attempt and will remain using the same RA Slot if further retransmissions are needed. Upon initial access, a device selects a RA Slot and waits for the Msg. 2 to get the current status of the CRQ. If there is an ongoing contention in the RA Slot $(R Q>1)$, new devices are not allowed to enter

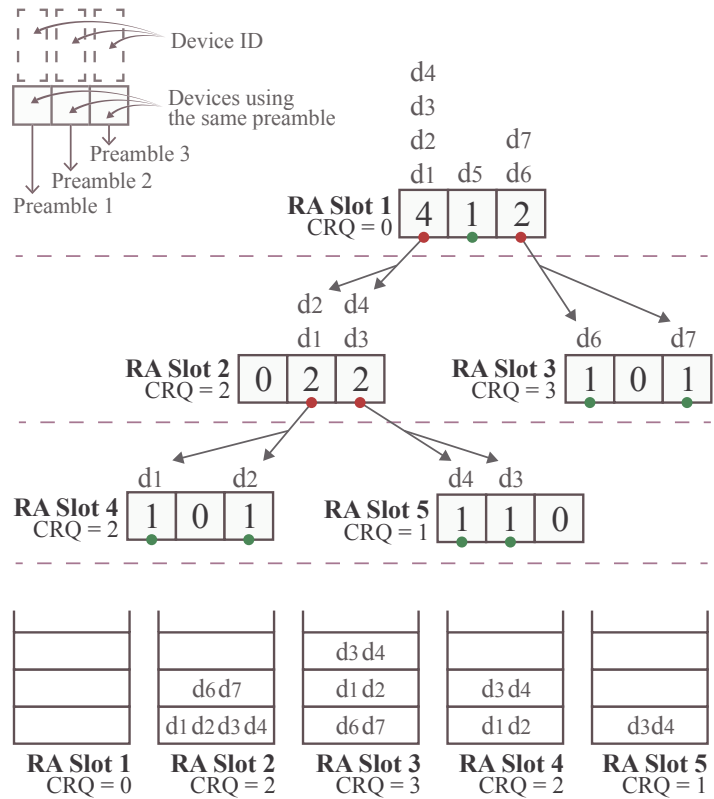

Fig. 4. Tree-splitting algorithm and CRQ behavior in the collision resolutions. For this example, 3 preambles are available on each RA slot. The number inside every preamble square denotes the number of devices that transmit the same preamble. On the lower part, a representation of the CRQ is depicted.

until all collisions have been solved. Therefore, the device will not transmit in the next RA Slot and repeats the process until there are no further collisions.

If a free RA Slot is found, where no collisions are being resolved, the device will send a preamble on the next occurrence of the RA Slot and wait for the corresponding Msg. 2. Three states must be provided on the Msg. 2 and the devices will do as follows:

1) Empty state: no preamble was received. The device will increase by one the preamble retransmission counter and reenter the CRQ.

2) Collision state: the device will increase by one the preamble retransmission counter and reenter the CRQ.

3) Success state: the device will decode the RAR and proceed to the transmission of Msg. 3.

\section{B. DQ feedback implementation in Msg. 2}

In order to integrate DQ in the initial access procedure, the RAR needs to be extended to accommodate the feedback information. Msg. 2 is a MAC PDU composed of a variable size header and zero or more RAR payloads. According to [15], the header consists of one or more subheader of the following types:

- RAPID Subheaher (Fig. 5(a)): RAPID stands for Random Access Preamble Identifier, which correspond to the preamble number. There will be one RAPID subheader for each successfully received preamble.

- BI Subheader (Fig. 5(b)): there is at the most one of these subheaders per Msg. 2 and it contains the BI parameter.

The subheader's fields are shown in Fig. 5 and correspond to: - E: extension bit, it means a subheader is attached after. 
Standard RA procedure with ACB vs. DQ-based RA procedure
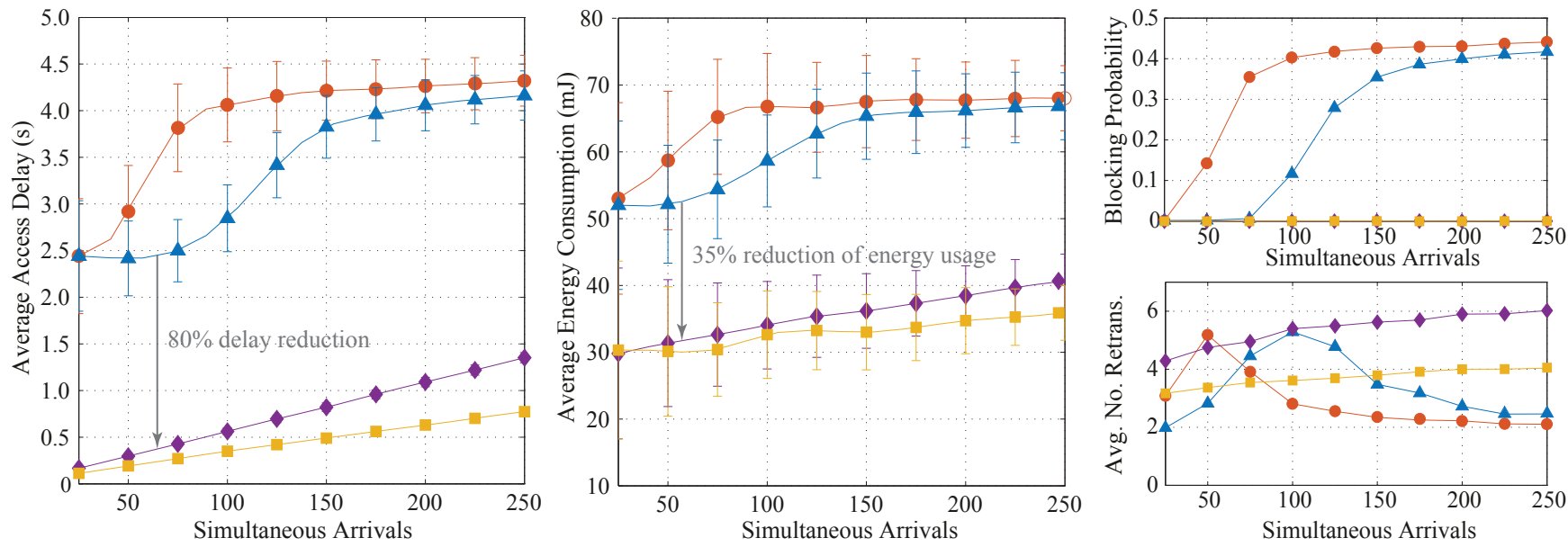

$\begin{array}{llll}\longrightarrow \text { Standard RA procedure with ACB with } 3 \text { orthogonal preambles. } & \longrightarrow \text { DQ-based RA procedure with } 3 \text { orthogonal preambles. } \\ \longrightarrow \quad \text { Standard RA procedure with ACB with } 6 \text { orthogonal preambles. } & - \text { DQ-based RA procedure with } 6 \text { orthogonal preambles. }\end{array}$

Fig. 7. Comparative between the standard RA procedure with ACB and the proposed DQ-based Ra procedure using the same number of orthogonal preambles. The selection of the number of preambles on each case is based on the relation provided in Fig. 3 .

1) Comparison using the same amount of preambles. For this test, the cases or cell radii 39.01 and $16.13 \mathrm{~km}$ are considered, corresponding to 3 and 6 as the maximum number of orthogonal preambles that can be generated from the same root sequence.

2) Comparison using different number of preambles. For this test, the cases of 5.68 and 3.68 cell radii are considered; for these cases, the standard RA procedure is capable of using 18 and 26 orthogonal preambles while the DQ-based RA procedure will be limited to 6 and the maximum number of available preambles, as described in Section IV.

In Fig. 7, the results for the first test can be appreciated. The limitations of the standard RA procedure are evident when the number of preambles available is low, having a direct negative impact on the access delay and energy consumption. Moreover, the blocking probability reaches unacceptable rates, above $40 \%$. This could be prevented on the current standard by either increasing the $\mathrm{BI}$, increasing the barring time or decreasing the barring rate. Nonetheless, these changes will result in even higher access delays. The simple CRQ implementation shows an effective improvement for these cases. As highlighted in Fig. 7, if we compare the performance of the standard procedure using 6 preambles and the DQ implementation using half of these preambles, we achieve at least $80 \%$ average access delay reduction and 35\% reduction on the average energy consumption, while maintaining a consistently low blocking probability, regardless of the number of initial simultaneous access attempts. The largest gains are achieved when comparing both procedures using 3 preambles for 100 simultaneous arrivals, reaching a $85 \%$ reduction on the access delay and $54 \%$ reduction on energy consumption for cells with radius larger than $39 \mathrm{~km}$.

The comparison results for the second test are shown in Fig. 8. For a $5.68 \mathrm{~km}$ cell radius it is possible to generate up to 18 orthogonal preambles from a single root sequence.
Nevertheless, the delay and energy consumption can still be improved with only 3 preambles by implementing the DQbased RA procedure. For a smaller cell radius of $3.68 \mathrm{~km}$, up to 26 preambles are used in the standard RA procedure. Here, the performance is better than using the DQ-based RA procedure with 3 preambles. However, the access delay is very similar compared to the DQ-based RA procedure using 6 preambles, but with a slightly higher energy consumption. The current standard can achieve a desirable performance under this configurations up to 200 simultaneous arrivals, at which point the blocking probability starts to increase. As previously explain, the current procedure cannot mitigate such increase without affecting the access delay or energy consumption.

If more than 26 preambles are available (cell radius smaller than $3.5 \mathrm{~km}$ ), it is evident that the standard procedure will allow a better access performance in terms of access delay. However, we have shown an alternative procedure that can provide better use to the available resources. Our current DQ implementation can only support up to 6 preambles, but further work should consider an extension of this idea, capable to use more preambles and extend the benefits presented in this paper.

\section{FinAl ASSESSMENT AND CONCLUSIONS}

In this work, we presented an extensive overview of the RA procedure in LTE in order to understand the relation between the cell radius and the number of available orthogonal preambles; this showed the resource limitations for cells with large radius. Moreover, we explained how the current RA procedure can only accommodate high number of devices by increasing the backoff parameters for preamble retransmissions, resulting in increased access delay and energy consumption.

According to these limitations, we presented an alternative procedure based on a tree-splitting algorithm and a distributed queue which is capable of reducing the access delay, while reducing the energy consumption and maintaining a low 
Standard RA procedure with ACB vs. DQ-based RA procedure
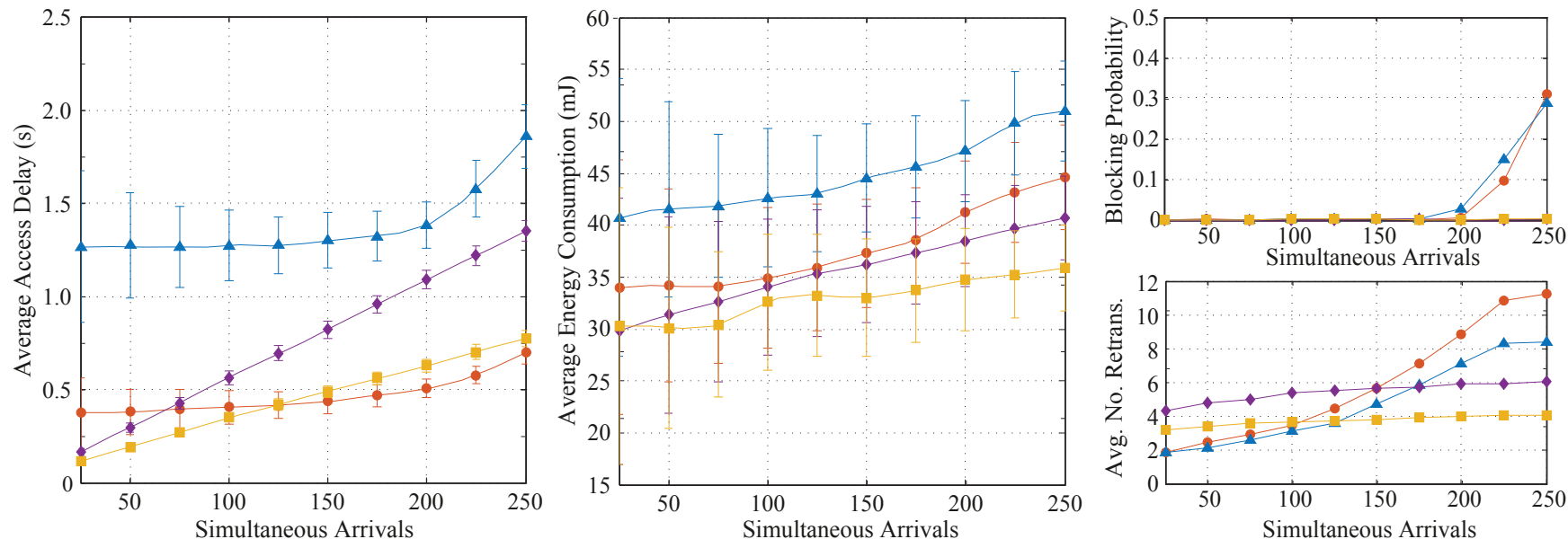

$\begin{array}{llll}\longrightarrow \text { Standard RA procedure with ACB - } 26 \text { orthogonal preambles. } & \longrightarrow \text { DQ-based RA procedure - } 3 \text { orthogonal preambles. } \\ \longrightarrow \quad \text { Standard RA procedure with ACB - } 18 \text { orthogonal preambles. } \quad \longrightarrow \text { DQ-based RA procedure }-6 \text { orthogonal preambles. }\end{array}$

Fig. 8. Comparative between the standard RA procedure with ACB and the proposed DQ-based Ra procedure for cell radii of 5.68 and 3.68 km. The selection of the number of preambles on each case is based on the relation shown Fig. 3.

blocking probability for an increasing amount of simultaneous access attempts. The solution can be implemented with simple modifications to the current standard and it is specially interesting for large cells, showing improved performance above $5 \mathrm{~km}$ of radius. The largest gains presented reach a $85 \%$ reduction on the access delay and 54\% reduction on energy consumption for cells with $39 \mathrm{~km}$ radius.

The implementation presented in this work is bounded to a maximum of 6 preambles and further work should target an extension of these principles in order to make use of all the available preambles and compare the performance with the current standard.

\section{ACKNOWLEDGMENT}

This work has been partially funded by Wireless@KTH, the EIT ICT Labs project EXAM, ADVANTAGE (FP7-607774), P2P-SMARTEST (H2020-646469) and the Catalan Government under grant (2014-SGR-1551).

\section{REFERENCES}

[1] 3GPP TR 22.868 V8.0.0, "Study on Facilitating Machine to Machine Communication in 3GPP Systems," March 2007.

[2] 3GPP TR 37.868 V11.0.0, "Study on RAN Improvements for Machinetype Communications," September 2011.

[3] 3GPP TSG RAN WG2 \#69bis R2-102296, "RACH intensity of Time Controlled Devices," Beijing, China, April 2010.

4] B. Yang, G. Zhu, W. Wu, and Y. Gao, "M2m access performance in ltesystem," Transactions on Emerging Telecommunications Technologies, vol. 25, no. 1, pp. 3-10, 2014.

[5] A. Laya, L. Alonso, and J. Alonso-Zarate, "Is the Random Access Channel of LTE and LTE-A Suitable for M2M Communications? A Survey of Alternatives," Communications Surveys Tutorials, IEEE, vol. 16, no. 1, pp. 4-16, First 2014.

[6] W. Xu and G. Campbell, "A Near Perfect Stable Random Access Protocol for a Broadcast Channle," in IEEE Proc. ICC 92, vol. 1, 1992 , pp. 370-374.
[7] — , "DQRAP, A Distributed Queueing Random Access Protocol for a Broadcast Channel," in Computer Commun. Mag., vol. 23, no. 4, Oct. 1993, pp. 370-374.

[8] L. Alonso, R. Agustí, and O. Sallent, “A Near-Optimum MAC Protocol Based on the Distributed Queueing Random Access Protocol (DQRAP) for a CDMA Mobile Communication System," in IEEE Journal on Selected Areas in Communications, vol. 18, no. 9, Sep. 2000, pp. 17011718

[9] B. Otal, L. Alonso, and R. Agustí, "Design and analysis of a cellular mobile communications system based on DQRAP/CDMA MAC protocol," in IEEE Electronic Letters, vol. 38, no. 3, Jan. 2002, pp. 138-139.

[10] E. Kartsakli, J.Alonso-Zárate, C. Verikoukis, and L. Alonso, "CrossLayer Enhancement for WLAN Systems with Heterogeneous Traffic based on DQCA," in IEEE Communications Magazine, vol. 46, no. 6 , Jun 2008, pp. 60-66.

[11] J. Alonso-Zárate, E. Kartsakli, L. Alonso, and C. Verikoukis, "Performance Analysis of a Cluster-Based MAC Protocol for Wireless Ad Hoc Networks," in EURASIP Journal on Wireless Communications and Networking, vol. 2010, 2010.

[12] B. Otal, C. Verikoukis, and L. Alonso, "Highly Reliable Energy-saving MAC for Wireless Body Sensor Networks in Healthcare Systems," in IEEE Journal on Selected Areas in Communications, Special Issue on Wireless and Pervasive Communications in Healthcare, vol. 27, no. 4 , May 2009, pp. 553-565.

[13] Sesia, S. and Baker, M. and Toufik, I., LTE - The UMTS Long Term Evolution: From Theory to Practice. Wiley, 2011, pp. 421-456.

[14] 3GPP TS 36.331 V10.5.0, "Evolved Universal Terrestrial Radio Access (E-UTRA); Radio Resource Control (RRC),” March 2012

[15] 3GPP TS 36.321 V9.3.0, "Evolved Universal Terrestrial Radio Access (E-UTRA); Medium Access Control (MAC)," June 2010.

[16] J. D. C. Chu, "Polyphase Codes with Good Periodic Correlation Properties," in IEEE Trans. on Information Theory, vol. 18, July 1972, pp. 531-532.

[17] R. Frank, S. Zadoff, and R. Heimiller, "Phase Shift Pulse Codes With Good Periodic Correlation Properties," in IEEE Trans. on Information Theory, vol. 8, October 1962, pp. 381-382.

[18] 3GPP TS 36.211 V10.4.0, "Evolved Universal Terrestrial Radio Access (E-UTRA); Physical Channels and Modulation,” December 2011.

[19] S. Hamalainen, H. Sanneck, and C. Sartori, LTE Self-Organising Networks (SON): Network Management Automation for Operational Efficiency. Wiley, 2012.

[20] T. Tirronen, A. Larmo, J. Sachs, B. Lindoff, and N. Wiberg, "Machineto-machine communication with long-term evolution with reduced device energy consumption," Transactions on Emerging Telecommunications Technologies, vol. 24, no. 4, pp. 413-426, 2013. 